Ms Hazelbaker and her work on tongue-tie. However, we also stand by the integrity of our study.

Diane J. Madlon-Kay, MD, MS

Lori A. Ricke, MD

Regions Family and Community Medicine Residency

Program

Nancy J. Baker, MD

Department of Community Medicine and Family

Health

University of Minnesota Medical School

St. Paul

\section{Postvasectomy Semen Analysis}

To the Editor: I read with interest the article by Christensen and Maples ${ }^{1}$ addressing postvasectomy semen analysis and the low compliance with instructions to confirm azoospermia. It prompted the following questions-when and how often is semen analysis required after a vasectomy, and is it ever necessary after a vasectomy? Furthermore, is it necessary to send the excised ends of the vas deferens for histopathological evaluation?

A review of the literature suggests that there is no definite agreement regarding the timing or the frequency of postvasectomy semen analysis. All ejaculates contain potentially fertile spermatozoa immediately after vasectomy, which become rapidly immobile within a few days, and usually by 3 weeks following the procedure. ${ }^{2}$ The British Andrology Society guidelines requires patients to wait 4 months or have at least 24 ejaculations before semen analysis. ${ }^{3}$ The society also recommends that patients not ejaculate for 48 hours prior to collection, collect semen by masturbation directly into the container, avoid condoms, and deliver the semen within an hour of collection. ${ }^{3}$ The World Health Organization has different recommendations-one or 2 semen analyses after 12 weeks or 15 ejaculations. ${ }^{4}$

Azoospermia proven on a single semen analysis at 3 months is probably sufficient grounds for discontinuing other methods of contraception. ${ }^{5}$ Further semen analyses should be required only if live sperm are present. Nonmotile sperm are probably not an indication for checking further semen samples. ${ }^{6}$ Patient compliance is good if they are required to submit only one sample for analysis but decreases significantly when they are asked to provide a second sample..$^{5}$

I suspect that postvasectomy semen analysis, though logical, is simply not necessary. Perhaps many patients (nearly $40 \%$ of my 360 ) realize this instinctively, wait the specified 3 to 4 months or, in many instances, 12 to 15 ejaculations, before commencing unprotected intimacy with their partners. A small percentage will undoubtedly have unintended issues, but humans gamble on success, and change will be difficult.

There is also no uniformity regarding histologic evaluation of the vasectomy specimens. One series from the United Kingdom showed that only three fourths of the surgeons followed this practice. ${ }^{6}$ Provided that the vasa are confidently identified and sectioned, routinely evaluating specimens just adds to the cost. Of the patients requesting vasectomy in my practice, most pay for the procedure themselves, and they can ill-afford this added expense. Hence, I have tended to preserve the vas deferens specimens until azoospermia is established at 3 months, or for 1 year, after which time the specimens are discarded because of space constraints. This is explained to the patients before the vasectomy. I suspect many family physicians practicing in rural communities do the same.

K. Ramakrishnan, MD

Department of Family and Preventive Medicine University of Oklahoma Health Sciences Center Oklahoma City

\section{References}

1. Christensen RE, Maples DC Jr. Postvasectomy semen analysis: are men following up? J Am Board Fam Pract 2005; $18: 44-7$.

2. Edwards IS. Earlier testing after vasectomy, based on the absence of motile sperm. Fertil Steril 1993;59:431-6.

3. Hancock P, McLaughlin E. British Andrology Society guidelines for the assessment of post vasectomy semen samples. J Clin Pathol 2002;55:812-6.

4. Technical and managerial guidelines for vasectomy services. Geneva: World Health Organization; 1988. p. 67.

5. Badrakumar C, Gogoi NK, Sundaram SK. Semen analysis after vasectomy: when and how many? BJU Int 2000;86: 479-81.

6. Sivardeen KA, Budhoo M. Post vasectomy analysis: call for a uniform evidence-based protocol. Ann R Coll Surg Engl 2001;83:177-9.

\section{Author's Reply}

To the Editor: Dr Ramakrishnan has raised some very good points. There is no absolute protocol for the number or timing of postvasectomy semen analyses. He suggests that a single 3 -month postvasectomy semen analysis would probably suffice, which seems reasonable. My research, however, indicates that less than half the men returned at 3 months (25\%) than returned for the 6-week check (58\%). Because a semen analysis is the only way to know that one has achieved azoospermia — and that is the purpose of the vasectomy - then this noninvasive sampling is logical.

Our study followed Denniston and Pfenninger, ${ }^{1}$ which suggested customary postoperative care, with the exception that we also encouraged a 12 -month postoperative semen check, in which only $8 \%$ of men participated. I also agree that because $42 \%$ of my patients did not return for ANY postvasectomy semen analysis, there are a significant number of risk-takers getting a vasectomy. It has been our practice not to routinely send specimens of excised vas deferens to pathology, because it just incurs more cost and does not determine the success of the vasectomy.

Ronald E. Christensen, MD Independence Park Medical Associates Anchorage, AK 